Reprint Number 3

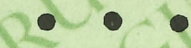

Engineering Experiment Station

Columbia, Missouri

\title{
STEADY-STATE WAVES \\ ON TRANSMISSION LINES
}

D. L. WAIDELICH * Professor of Electrical Engineering

Reprinted From Trans. AIEE, Vol. 69

1950 
The Engineering Experiment Station was organized in 1909 as a part of the College of Engineering. The staff of the Station includes all members of the Faculty of the College of Engineering, together with Research Assistants supported by the Station Funds.

The Station is primarily an engineering research institution engaged in the investigation of fundamental engineering problems of general interest, in the improvement of engineering design, and in the development of new industrial processes.

The Station desires particularly to co-operate with industries of Missouri in the solution of such problems. For this purpose, there is available not only the special equipment belonging to the Station but all of the equipment and facilities of the College of Engineering not in immediate use for class instruction.

Inquiries regarding these matters should be addressed to

The Director,

Engineering Experiment Station

University of Missouri

Columbia, Missouri

\section{THE UNIVERSITY OF MISSOURI BULLETIN}

VOLUME 52, NUMBER 16 ENGINEERING EXPERIMENT STATION REPRINT SERIES NO. 3

Published by the University of Missouri at Room 102, Building T-3, Columbia, Missouri. Entered as second-class matter, January 2, 1914, at the postoffice at Columbia, Missouri, under the Act of Congress of August 24, 1912. Issued three times a month. .-300

JUNE 1, 1951 


\section{Steady-State $W$ aves on Transmission Lines}

\author{
D. L. WAIDELICH \\ MEMBER AIEE
}

$\mathbf{T}$ HE increased use of nonsinusoidal waves on transmission lines such as, for example, the location of faults on the lines $^{1}$ by means of repeated pulses, has made it desirable to consider how the response of the lines to the applied nonsinusoidal waves may be obtained. The transient response of the line has been presented in a number of previous papers $^{2,3}$ but the steady-state response seems to have been neglected. It is the purpose of this paper to consider various methods of obtaining the steady-state response of transmission lines to applied nonsinusoidal waves and also to give several experimental results for purposes of comparison. These methods apply as well to plane electromagnetic waves propagating in a good dielectric which is isotropic.

In making the mathematical analysis it is assumed that the frequencies are sufficiently high so that certain simplifications may be made. For example, in the operational expression for the characteristic impedance $Z_{0}$ of the transmission line

$$
\begin{aligned}
Z_{0}= & \sqrt{(R+L p) /(G+C p)} \\
& =\sqrt{\frac{L}{C}\left[1+\frac{1}{p}\left(\frac{R}{2 L}-\frac{G}{2 C}\right)+\ldots\right]}
\end{aligned}
$$

where $R, L, G, C$ are the resistance, the inductance, the conductance, and the capacitance of the line for a unit length, it is assumed that only the first term in

Paper 50-244, recommended by the AIEE Basic Sciences Committee and approved by the AIEE Technical Program Committee for presentation at the AIEE Fall General Meeting, Oklahoma City, Okla., October 23-27, 1950. Manuscript submitted March 13, 1950; made available for printing August 30, 1950

D. L. WAIDELICH is with the University of Missouri, Columbia, Mo. the series is necessary. From this point on it will then be assumed that $Z_{0}=$ $\sqrt{L / C}$. Similarly for the propagation constant $\gamma$ the operational expression is

$$
\begin{array}{r}
\gamma=\sqrt{(R+L p) G+C p)} \\
=p \sqrt{L C}\left[1+\frac{1}{p}\left(\frac{R}{2 L}-\frac{G}{2 C}\right)-\right. \\
\left.\frac{1}{8 p^{2}}\left(\frac{R}{L}-\frac{G}{C}\right)^{2}+\ldots\right]
\end{array}
$$

and only the first two terms in the series will be used so that

$\gamma=(p / V)+a$

where $V=1 / \sqrt{L C}=$ velocity of propagation of the waves and

$a=\frac{R}{2} \sqrt{\frac{C}{L}}+\frac{G}{2} \sqrt{\frac{L}{C}}=$ attenuation constant

To show how the various methods may be used to determine the steady-state waves appearing on a transmission line, a particular example will be employed. This example is that of a line with a square-wave generator connected to one end while the other end is open-circuited The voltage $e_{R}$ at the open end is to be determined. The generator is assumed to have zero internal impedance, and the generated voltage $e_{S}$ is shown in Figure 1. The steady-state transform ${ }^{4} E_{S}$ of the generated voltage is

$E_{n}=\frac{E}{p}\left(1-\epsilon^{-P T / 2}\right)^{2}$

The steady-state transform $E_{R}$ of the voltage at the open end of the line is

$E_{R}=\frac{E_{s}}{\cosh \gamma l}$

where $l$ is the length of the line. The voltage is then
$\varepsilon_{R}=\frac{1}{2 \pi j} \int_{W} \frac{\epsilon^{p t} E\left(1-\epsilon^{-p^{T / 2}} d p\right.}{p\left(1-\epsilon^{-p^{T}}\right) \cosh [(p l / V)+a l)}$

where $W$ is a certain path of integration $^{4}$ in the $p$-plane. If the substitutions $p^{\prime}=p T, \beta=(l / V T), \alpha=a l$ and $\theta=(t / T)$ are made in equation 6 ,

$\frac{e_{R}}{E}=\frac{1}{2 \pi j} \int_{W} \frac{\epsilon^{p^{\prime} \theta}\left(1-\epsilon^{-p^{\prime} / 2}\right)^{2} d p^{\prime}}{p^{\prime}\left(1-\epsilon^{-p^{\prime}}\right) \cosh \left(p^{\prime} \beta+\alpha\right)}$

The primes now will be omitted.

The first method of evaluating the integral of equation 7 uses the poles within the path $W$, sometimes called the driving function or "voltage" poles, and leads to the usual Fourier series for the voltage $e_{R}$. These poles are $p= \pm j 2 \pi n$ where $n$ is a positive integer or zero. When the residues at each one of these poles are added, the result is

$\frac{e_{R}}{E}=\sum_{n=1}^{\infty} Y_{n} \sin (2 n \pi \theta-\delta)$

which is summed over all odd integral values of $n$ and in which

$Y_{n}=4 / n \pi \sqrt{\sinh ^{2} \alpha+\cos ^{2} 2 n \pi \beta}$

and

$\delta=\tan ^{-1} \cdot[(\tanh \alpha)(\tan 2 n \pi \beta)]$

The even harmonic voltages are absent in $e_{R}$ since there are none in the applied square wave of voltage. The result of equation 8 is useful in determining the amount of harmonic voltages present, but is of very little use in determining the actual waveforms present except in some very special cases. One such special case is that for $\alpha=0$ and $\beta=1 / 6$ which means that the losses in the transmission line are zero and that a wave on the line travels the length of the line in one-sixth of the period of the applied square wave. From equation 8 the open end voltage is

$$
\begin{aligned}
& \frac{e_{R}}{E}=2\left[\frac{4}{\pi} \sum_{n=1}^{\infty} \frac{\sin 2 n \pi \theta}{n}\right]- \\
& {\left[\frac{4}{\pi} \sum_{n=1}^{\infty} \frac{\sin 6 n \pi \theta}{n}\right] }
\end{aligned}
$$




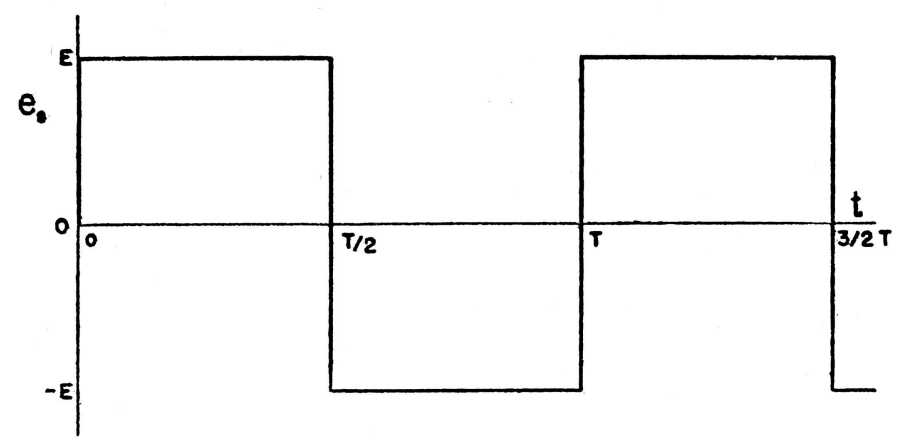

Figure 1. The square-wave voltage $e_{S}$ of the generator

which are summed over all odd integral values of $n$. The first series of equation 9 is a square wave of amplitude $2 E$ and period $T$, while the second is a square wave of amplitude $E$ and period $(T / 3)$. The resulting wave is shown in Figure 2, and this may be called the sum function of the series of equation 9 .

For circuits with concentrated parameters the sum function of the Fourier series may be obtained without difficulty by using the poles outside the path $W$, sometimes called the system-function or "impedance" poles. In most circuits these poles are finite in number and thus lead to an expression with a finite number of terms. This approach was tried as the second method of evaluating the integral of equation 7 . The poles are $-(\alpha / \beta) \pm j$ $(m \pi / 2 \beta)$, where $m$ is an odd positive integer. Since there are again an infinite number of poles, the resulting expression ${ }^{4}$ is an infinite series somewhat resembling a Fourier series. When $0<\theta<1 / 2$, the voltage at the open-circuited end is

$\frac{e_{R}}{E}=\frac{1}{\cosh \alpha}+\sum_{m=1}^{\infty} \frac{(-1)^{\frac{m+1}{2}} 4 \epsilon^{-\sigma \theta}}{\beta\left(\sigma^{2}+\mu^{2}\right)} A_{m}$

in which

$$
m=\text { an odd positive integer }
$$$$
A_{m}=\frac{\begin{array}{c}
\sigma\left[\sin \mu \theta+\epsilon^{-\sigma / 2} \sin \mu(\theta-1 / 2)\right]+ \\
\mu\left[\cos \mu \theta+\epsilon^{-\sigma / 2} \cos \mu(\theta-1 / 2)\right]
\end{array}}{\left[1+2 \epsilon^{-\sigma / 2} \cos (\mu / 2)+\epsilon^{-\sigma}\right]}
$$

$\sigma=\alpha / \beta$

$\mu=m \pi / 2 \beta$

Again this is useful in determining waveforms only in some special cases such as the one used in the preceding paragraph. If $\alpha=0$ and $\beta=1 / 6$, the result is a Fourier series similar to equation 9 but not the same series.

$$
\begin{aligned}
\frac{e_{R}}{E}=1+\frac{4}{\pi} & \sum_{m=1}^{\infty} \frac{\sin 3 \pi m \theta}{m}- \\
& \frac{4}{\pi} \sum_{m=1}^{\infty}(-1)^{\frac{m-1}{2}} \frac{\cos 3 \pi m \theta}{m}
\end{aligned}
$$

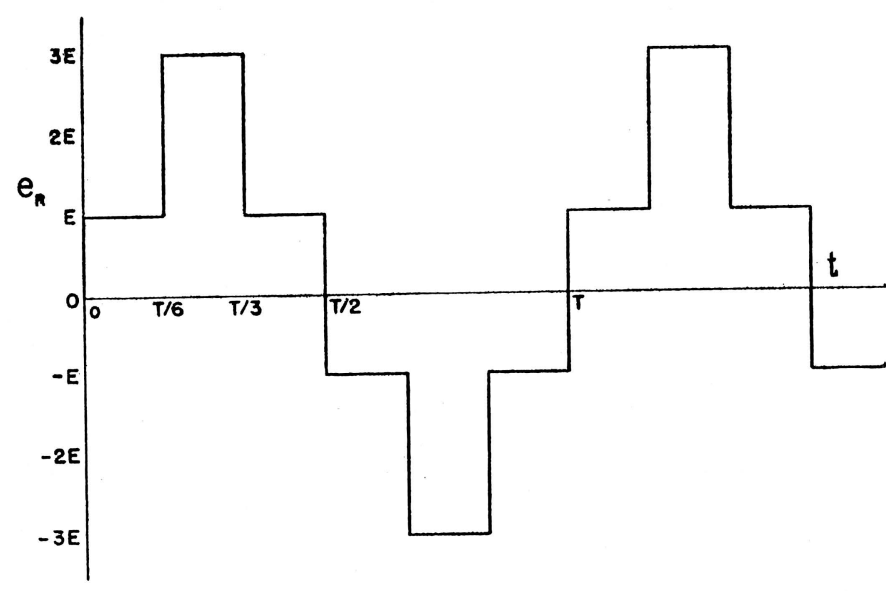

Figure 2. The open-end voltage $e_{R}$ of the transmission line with no attenuation $(\alpha=0)$

in which $m$ is an odd positive integer. The second term of equation 11 is a square wave of amplitude $E$ and period $(2 T / 3)$, while the third term is a square wave of the same amplitude and period but shifted in phase by $(T / 6)$. When the three terms of equation 11 are added, the result is again the wave of Figure 2.

The third method involves the use of steady-state convolution or "faltung" integrals. ${ }^{5}$ Let $A(t)$ be the voltage at the open end of the line when a unit voltage is suddenly applied ${ }^{2}$ to the generator end of the line, and $A(t)=A(\infty)+R(t)$ where $A(\infty)$ is a constant approached by $A(t)$ as $t$ becomes very large and then $R(t)$ approaches zero as $t$ becomes large. The form of the steady-state convolution integral to be used is:

$e_{R}=A(\infty) e_{8}(t)+\frac{d}{d t} \int_{0}^{T} e_{s}(t-\tau) P(\tau) d \tau$

where

$P(t)=\sum_{n=0}^{\infty} R(t+n T)$

and $n$ is a positive integer or zero. In the example at hand, from $\theta=0$ to $\theta=\beta$, $A(t)=0$, and from $\theta=m \beta$ to $(m+2) \beta$,

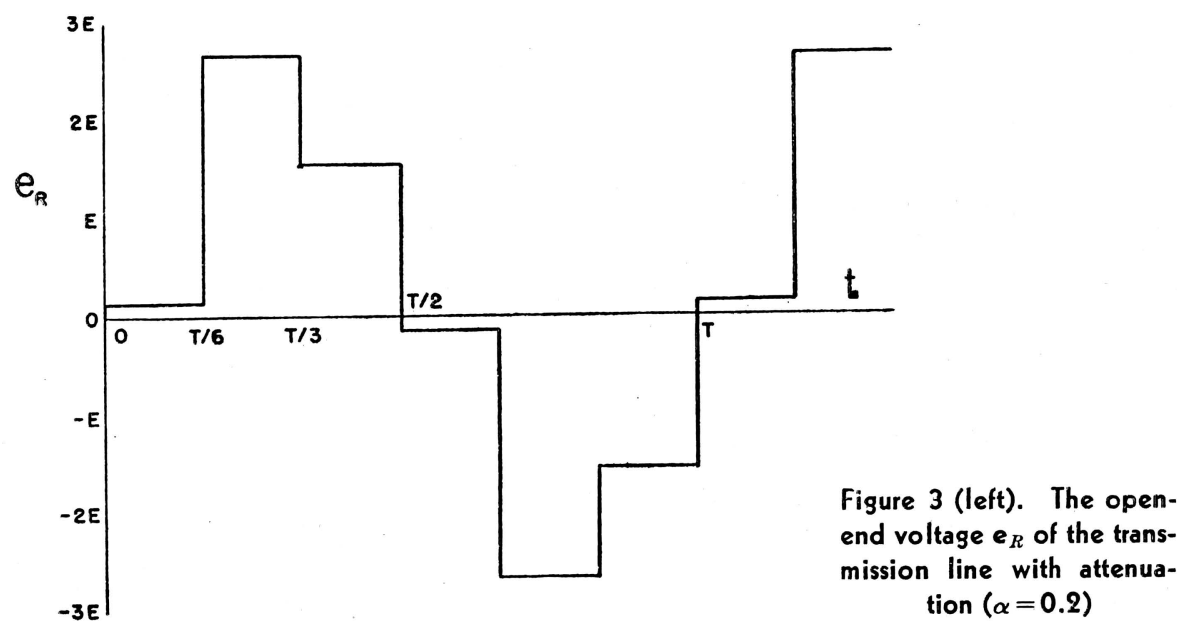

where $m$ is an odd positive integer,

$A(t)=2 \epsilon^{-\alpha}\left[\frac{1+(-1)^{\frac{m-1}{2}} \epsilon^{-(m+1) \alpha}}{1+\epsilon^{-2 \alpha}}\right]$

Then

$A(\infty)=2 \epsilon^{-\alpha} /\left(1+\epsilon^{-2 \alpha}\right)$

and also from $\theta=0$ to $\theta=\beta, R(t)=-2 \epsilon^{-\alpha}$ / $\left(1+\epsilon^{-2 \alpha}\right)$, and from $\theta=m \beta$ to $\theta=(m+$ 2) $\beta$, where $m$ is an odd positive integer

$R(t)=(-1)^{\frac{m-1}{2}} \frac{2 \epsilon^{-(m+2) \alpha}}{1+\epsilon^{-2 \alpha}}$

Then for the special case of $\beta=1 / 6$, from $\theta=0$ to $\theta=1 / 6$,

$$
P(t)=\frac{-2 \epsilon^{-\alpha}}{\left(1+\epsilon^{-2 \alpha}\right)\left(1+\epsilon^{-6 \alpha}\right)}
$$

and from $\theta=m / 6$ to $\theta=(m+2) / 6$ where $m$ is an odd positive integer

$P(t)=(-1)^{\frac{m-1}{2}} \frac{2 \epsilon^{-(m+2) \alpha}}{\left(1+\epsilon^{-2 \alpha}\right)\left(1+\epsilon^{-6 \alpha}\right)}$

With the aid of equations 12,14 , and 16 and in the interval from $\theta=0$ to $\theta=1 / 6$ :

$e_{R}=\frac{2 E\left(-\epsilon^{-\alpha}+\epsilon^{-3 \alpha}+\epsilon^{-5 \alpha}\right.}{\left(1+\epsilon^{-6 \alpha}\right)}$ 


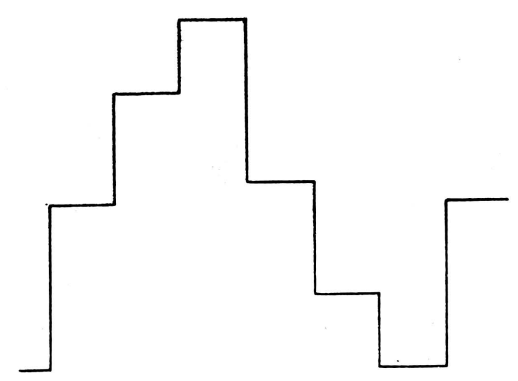

Figure 4. The calculated output voltage of the line for an applied square wave

from $\theta=1 / 6$ to $\theta=1 / 3$

$e_{R}=\frac{2 E\left(\epsilon^{-\alpha}+\epsilon^{-3 \alpha}+\epsilon^{-5 \alpha}\right)}{\left(1+\epsilon^{-6 \alpha}\right)}$

and from $\theta=1 / 3$ to $\theta=1 / 2$

$e_{R}=\frac{2 E\left(\epsilon^{-\alpha}+\epsilon^{-3 \alpha}-\epsilon^{-5 \alpha}\right)}{\left(1+\epsilon^{-6 \alpha}\right)}$

The values of $e_{R}$ for the second half cycle from $\theta=1 / 2$ to $\theta=1$ are the negatives of those given in equation 17 . When $\alpha=0$, the receiving end voltage as calculated from equation 17 is again the wave of Figure 2. For a line with losses the wave is not quite so symmetrical as is shown in Figure 3 for the case of $\alpha=0.2$.

The fourth method depends upon an extension and summation of the various waves obtained from the response $A(t)$ to a unit voltage. Consider

$A(t)=\frac{1}{2 \pi j} \int_{W_{1}} \frac{\epsilon^{p \theta}\left(1-\epsilon^{-p / 2}\right)^{2} d p}{p\left(1-\epsilon^{-p}\right) \cosh (p \beta+\alpha)}$

where $W_{1}$ is the usual path of integration for the inverse Laplacian transform and is to the right and parallel to the imaginary axis. Equation 18 should be compared with equation 7 . By use of the series:

$$
\begin{aligned}
\frac{\left(1-\epsilon^{-p / 2}\right)^{2}}{1-\epsilon^{-p}}=1-2 \epsilon^{-p / 2}+2 \epsilon^{-p}- \\
2 \epsilon^{-3 p / 2}+\ldots
\end{aligned}
$$

and

$$
\begin{array}{r}
\frac{1}{\cosh (p \beta+\alpha)}=2 \epsilon^{-(p \beta+\alpha)}-2 \epsilon^{-3(p \beta+\alpha)}+ \\
2 \epsilon^{-5(p \beta+\alpha)}-\ldots
\end{array}
$$

the response to the unit voltage is

$$
A(t)=\sum_{m=1}^{\infty} A_{m}(t)
$$

where $m$ is an odd integer and

$$
\begin{array}{r}
A_{m}(t)=\frac{1}{2 \pi j} \int_{W_{1}}\left(-1^{\frac{m-1}{2}} 2 \epsilon^{-m(p \beta+\alpha)} \times\right. \\
{\left[1-2 \epsilon^{-p / 2}+2 \epsilon^{-p}-2 \epsilon^{-3 p / 2}+\ldots\right] \times} \\
\frac{\epsilon^{-p \theta}}{p} d p
\end{array}
$$

In the problem used as an example $\beta=$ $1 / 6$. Consider one of the terms in the series for $A(t)$, say

$$
\begin{aligned}
A_{3}(t)=(-2) \frac{1}{2 \pi j} \int_{W_{1}} \epsilon^{-3(p / 6+\alpha)} \times \\
\\
\quad\left[1-2 \epsilon^{-p / 2}+2 \epsilon^{-p}-\cdots\right] \frac{\epsilon^{p \theta}}{p} d p
\end{aligned}
$$

which represents a square wave beginning at $\theta=1 / 2$ and with an amplitude of $2 \epsilon^{-3} \alpha$. This square wave should be continued or extended back to $\theta=0$. Each one of the square waves for each of the $A_{m}$ 's should be extended back in a like manner to $\theta=0$. All of the square waves may then be summed to give the required steady-state output voltage $e_{R}$. The result is for $0<\theta<1 / 6$

$$
\begin{aligned}
\frac{e_{R}}{E} & =-2 \epsilon^{-\alpha}+2 \epsilon^{-3 \alpha}+2 \epsilon^{-5 \alpha}+2 \epsilon^{-7 \alpha}- \\
2 \epsilon^{-9 \alpha}-2 \epsilon^{-1 \alpha}-2 \epsilon^{-13 \alpha}+\ldots & \\
& =\frac{-2 \epsilon^{-\alpha}+2 \epsilon^{-3 \alpha}+2 \epsilon^{-5 \alpha}}{1+\epsilon^{-6 \alpha}}
\end{aligned}
$$

for $1 / 6<\theta<1 / 3$

$\frac{e_{R}}{E}=\frac{2 \epsilon^{-\alpha}+2 \epsilon^{-3 \alpha}+2 \epsilon^{-5 \alpha}}{1+\epsilon^{-6 \alpha}}$

for $1 / 3<\theta<1 / 2$

$\frac{e_{R}}{E}=\frac{2 \epsilon^{-\alpha}+2 \epsilon^{-3 a}-2 \epsilon^{-5 \alpha}}{1+\epsilon^{-6 \alpha}}$

These results are identical with equation 17 and will thus give the waves of Figures 2 and 3 .

\section{Experimental Results}

In the experimental work a squarewave generator with a high output im-

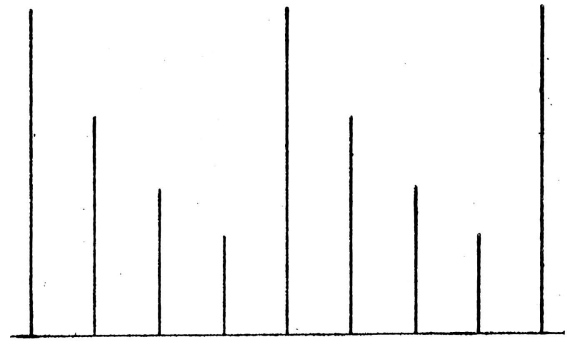

Figure 6. The calculated output voltage of the line for an applied pulse wave

pedance was used with a length of $R G$ $8 / U$ coaxial cable, and the voltage across the other end of the cable was observed with a high-impedance cathode-ray oscilloscope. For this purpose then both ends of the cable were effectively open circuited. Under these conditions the operational voltage at the receiving end is

$E_{R}=\frac{Z_{0} V_{g}}{R_{g} \sinh \gamma l}$

where $V_{g}$ is the transform of the generator voltage and $R_{g}$ is the output impedance (considered a pure resistance) of the generator. By the use of the fourth method given in the analysis section for $\beta=1 / 12$, it is found that for

$0<\theta<1 / 12, \frac{e_{R}}{E}=\frac{Z_{0}}{R_{0}}\left[\frac{-2 \epsilon^{-\alpha}-2 \epsilon^{-3 \alpha}-2 \epsilon^{-5 \alpha}}{1+\epsilon^{-6 \alpha}}\right]$

f or $1 / 12<\theta<1 / 4$

$\frac{e_{R}}{E}=\frac{Z_{0}}{R_{g}}\left[\frac{2 \epsilon^{-\alpha}-2 \epsilon^{-3 a}-2 \epsilon^{-5 \alpha}}{1+\epsilon^{-6 \alpha}}\right]$

for $1 / 4<\theta<5 / 12$

$\frac{e_{R}}{E}=\frac{Z_{0}}{\mathrm{R}_{0}}\left[\frac{2 \epsilon^{-\alpha}+2 \epsilon^{-3 \alpha}-2 \epsilon^{-5 \alpha}}{1+\epsilon^{-6 \alpha}}\right]$

for $5 / 12<\theta<1 / 2$

$\frac{e_{R}}{E}=\frac{Z_{0}}{R_{g}}\left[\frac{2 \epsilon^{-\alpha}+2 \epsilon^{-3 \alpha}+2 \epsilon^{-5 \alpha}}{1+\epsilon^{-6 \alpha}}\right]$

where $E$ is the peak value of the applied square wave.

The calculated receiving end voltage $e_{R}$ for $\alpha=0.2$ and $\beta=1 / 12$ is shown in Figure 4 , and the experimental wave form is that of Figure 5. Evidently the two

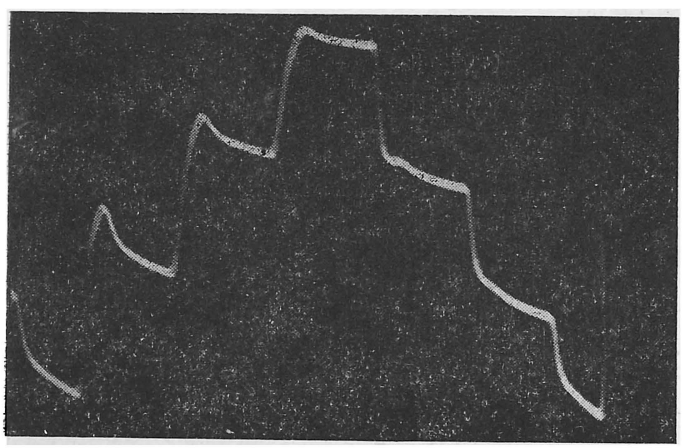

Figure 5 (left). The experimental output voltage of the line for an applied square wave

Figure 7 (right). The experimental output voltage of the line for an applied pulse wave

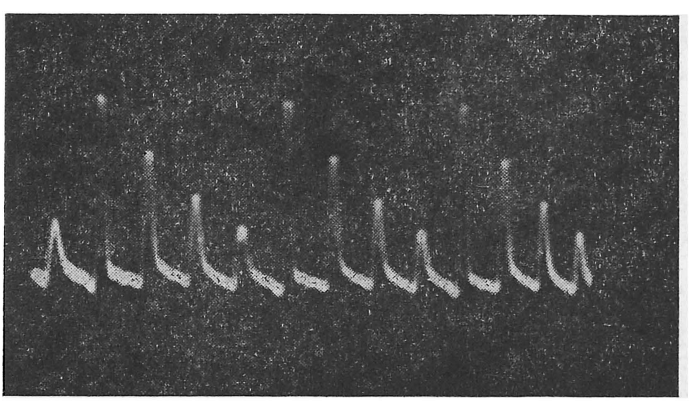


half waves of the applied square wave were not exactly equal because some dissymmetry is noticeable in the figure. Also some overshoot is visible and this might be caused in part by the characteristics of the oscilloscope used and also by the fact that the velocities, attenuations, and terminations of the cable are not those assumed in the analysis.

The action of repeated pulses ${ }^{6}$ on a transmission line also was studied. If $E$ is the transform of the applied repeated pulses, the output voltage $e_{R}$ will be calculated for a transmission line effectively open circuited at both ends. Again using the fourth method given in the analysis section for $\beta=1 / 8$, it is found that at $\theta=1 / 8$

$e_{R}=\frac{Z_{0} E}{R_{g} T}\left(\frac{2 \epsilon^{-\alpha}}{1-\epsilon^{-8 \alpha}}\right)$

at $\theta=3 / 8$

$e_{R}=\frac{Z_{0} E}{R_{g} T}\left(\frac{2 \epsilon^{-3 \alpha}}{1-\epsilon^{-8 \alpha}}\right)$

at $\theta=5 / 8$
$e_{R}=\frac{Z_{0} E}{R_{0} T}\left(\frac{2 e^{-5 \alpha}}{1-\epsilon^{-8 \alpha}}\right)$

at $\theta=7 / 8$

$e_{R}=\frac{Z_{0} E}{R_{0} T}\left(\frac{2 \epsilon^{-7 \alpha}}{1-\epsilon^{-8 \alpha}}\right)$

The output voltage would then be four pulses per period as shown in Figure 6 which was calculated for $\alpha=0.2$. The experimental wave form is shown in Figure 7 , and it was obtained using a pulse length of 0.5 microsecond and a pulse repetition rate of $84.3 \mathrm{kc}$ per second on a 957-foot length of $R G 8 / U$ coaxial cable. The velocity of propagation may be obtained from

$$
V=l / \beta T=1.97 \times 10^{8} \text { meters per second }(24)
$$

and the velocity factor, that is, the ratio of the velocity of the pulses on the line and the velocity of light in space, is 65.7 per cent. From equation 24 , at $\theta=n / 8$ where $n$ is $1,3,5$, or $7, e_{R}$ is proportional to $\epsilon^{-n \alpha}$. If $l n e_{R}$ is plotted versus $n$, the result should be a straight line of slope $(-\alpha)$. This was done with the waveform of Figure 7 , and a straight line resulted with a slope of -0.188 . The experimental value of $\alpha=0.188$, and the attenuation constant $a=(\alpha / l)=0.1964 \times 10^{-3}$ neper per foot or 0.1703 decibel per 100 feet of cable.

\section{References}

1. Pulse Echo Measurements on Thlbphone And Television Facrlitibs, L. G. Abraham, A: W. Lebert, J. B. Maggio, J. T. Schott. AIEE TransacLebert, J. B. Maggio, J. T. Schott. $A I B$, volume 66,1947 , pages 541-48.
tions,

2. Transformation Calculus and Electrical Transients (book), S. Goldman. Prentice-Hall, Inc., New York, N. Y., 1949, pages 286-315

3. Modern Operational Calculos (book), N. W. McLachlan. The MacMillan Company. New York, N. Y., 1948, pages 78-99.

4. The Steady-State Operational Calculus, D. L. Waidelich. Proceedings, Institute of Radio Engineers (New York, N. Y.), volume 34, 1946, pages $78-83$.

5. Strady State Currents of Electrical Networks, D. L. Waidelich. Journal of Applied Physics, (New York, N. Y.) volume 13, 1942, pages 706-12.

6. Response of Crrcuits to Steady-Statr Pulses, D. L. Waidelich. Proceedings, Institute of Radio Engineers (New York, N. Y.), volume 37, 1949 , pages $1396-1401$.

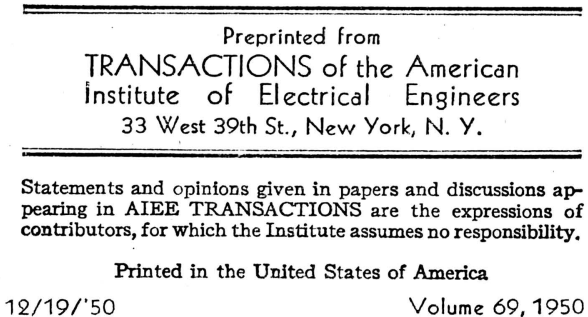




University of Missouri Libraries

University of Missouri

MU Engineering Experiment Station Series

Local Identifier Waidelich1951

Capture information

Date captured 2018 June

Scanner manufacturer Ricoh

Scanner model MP C4503

Scanning software

Optical resolution

Color settings

File types

$600 \mathrm{dpi}$

Grayscale, 8 bit; Color 24 bit

Tiff

Source information

Format

Content type

Book

Notes

Text

Digitized duplicate copy not retained in collection.

Derivatives - Access copy

\section{Compression LZW}

Editing software Adobe Photoshop

Resolution

Color

$600 \mathrm{dpi}$

File types

Grayscale, 8 bit; Color, 24 bit

Notes

Tiffs converted to pdf

Greyscale pages cropped and canvassed. Noise removed from background and text darkened.

Color pages cropped. 